\title{
Sensitization of gastric cancer cells to alkylating agents by glaucocalyxin $B$ via cell cycle arrest and enhanced cell death
}

This article was published in the following Dove Press journal:

Drug Design, Development and Therapy

22 August 2017

Number of times this article has been viewed

\author{
Muhammad Saif Ur \\ Rahman' \\ Ling Zhang ${ }^{2}$ \\ Lingyan $\mathrm{Wu}^{\prime}$ \\ Yuqiong $\mathrm{Xie}^{\mathrm{I}}$ \\ Chunchun $\mathrm{Li}^{\prime}$ \\ Jiang $\mathrm{CaO}^{\prime}$ \\ 'Clinical Research Center, \\ ${ }^{2}$ Cardiovascular Key Laboratory \\ of Zhejiang Province, The Second \\ Affiliated Hospital, Zhejiang \\ University School of Medicine, \\ Hangzhou, Zhejiang Province, \\ People's Republic of China
}

Correspondence: Jiang Cao

Clinical Research Center, The Second Affiliated Hospital, Zhejiang University School of Medicine, 88 jiefang Road, Hangzhou 310009 , Zhejiang Province, People's Republic of China

Tel +86 57| 873I 5202

Fax +86 57| 873। 520 I

Email caoj@zju.edu.cn
Abstract: Severe side effects are major problems with chemotherapy of gastric cancer (GC). These side effects can be reduced by using sensitizing agents in combination with therapeutic drugs. In this study, the low/nontoxic dosage of glaucocalyxin B (GLB) was used with other DNA linker agents mitomycin C (MMC), cisplatin (DDP), or cyclophosphamide (CTX) to treat GC cells. Combined effectiveness of GLB with drugs was determined by proliferation assay. The molecular mechanisms associated with cell proliferation, migration, invasion, cell cycle, DNA repair/replication, apoptosis, and autophagy were investigated by immunoblotting for key proteins involved. Cell cycle and apoptosis analysis were performed by flow cytometry. Reactive oxygen species level was also examined for identification of its role in apoptosis. Proliferation assay revealed that the addition of $5 \mu \mathrm{M}$ GLB significantly sensitizes gastric cancer SGC-7901 cells to MMC, DDP, and CTX by decreasing half-maximal inhibitory concentration $\left(\mathrm{IC}_{50}\right)$ by up to $75.40 \% \pm 5 \%, 45.10 \% \pm 5 \%$, and $52.10 \% \pm 5 \%$, respectively. GLB + drugs decreased the expression level of proteins involved in proliferation and migration, suggesting the anticancer potential of GLB + drugs. GLB + MMC, GLB + CTX, and GLB + DDP arrest the cells in $\mathrm{G}_{0} / \mathrm{G}_{1}$ and $\mathrm{G}_{1} / \mathrm{S}$ phase, respectively, which may be the consequence of significant decrease in the level of enzymes responsible for DNA replication and telomerase shortening. Combined use of GLB with these drugs also induces DNA damage and apoptosis by activating caspase/ PARP pathways and increased production of reactive oxygen species and increased autophagy in GC cells. GLB dosage sensitizes GC cells to the alkylating agents via arresting the cell cycle and enhancing cell death. This is of significant therapeutic importance in the reduction of side effects associated with these drugs.

Keywords: glaucocalyxin B, mitomycin C, cisplatin, cyclophosphamide, DNA linkers, side effects, gastric cancer

\section{Plain language summary}

Gastric cancer (GC) is one of the common malignancies worldwide. Although chemotherapy is often used in the treatment of the patients, it is also accompanied by severe side effects. The severity of these side effects can be reduced by using sensitizing agents that have the effect of decreasing the overall dose of the drugs to reduce side effects while maintaining their therapeutic effects. Our preliminary study showed that glaucocalyxin B (GLB) may have the potential to be a sensitizing agent for GC cells to cisplatin treatment. In the current work, we extensively investigated the effect and mechanisms for GLB as a sensitizing agent. We report that GLB can markedly sensitize GC cells to the alkylating drugs. The inclusion of low/nontoxic dosage of GLB in the treatment leads to cell cycle arrest and enhanced apoptosis/apoptosis. The novel findings of our work are of significant importance in the reduction of side effects associated with these drugs. 


\section{Introduction}

Gastric cancer (GC) is one of the common malignancies worldwide, with almost $70 \%$ of all the newly diagnosed GC cases from developing countries. ${ }^{1}$ Chemotherapy is one of the major modalities in the treatment of GC.

Alkylating agents are the oldest class of anticancer drugs used to play an imperative role in the treatment of various types of cancers. Mitomycin C (MMC), an alkylating agent used as a chemotherapeutic drug, can effectively inhibit tumor growth by damaging DNA. ${ }^{2}$ However, adverse renal cytotoxicity upon the clinical dosage of MMC has constrained its use in some patients. ${ }^{3}$

Currently, cisplatin (DDP) remains one of the most common, widely used, and effective anticancer alkylating agents, ${ }^{4}$ but the nonselective dissemination of DDP among normal and cancer tissues increases the prospect of doselimiting side effects including neurotoxicity, myelosuppression, and acute nephrotoxicity. ${ }^{5}$

Cyclophosphamide (CTX) is another alkylating agent frequently used as an anticancer drug, either alone or in combination with other medicines to treat several cancers. ${ }^{6}$ Like MMC and DDP, CTX also has a limitation due to the side effects. Sensitization of cancer cells to these alkylating drugs by decreasing the effective dosage when combined with certain agents may help to increase the tumoricidal activity and lessen the side effects associated with these drugs.

Rabdosia japonica ( $R$. japonica) is a perennial herb widely grown in East Asia. The aerial parts of $R$. japonica are being used as an antibacterial, anti-inflammatory, and anticancer agent in traditional medicine. ${ }^{7}$ Glaucocalyxin A and glaucocalyxin B (GLB) are major ent-kauranoid diterpenoids extracted from $R$. japonica shown to possess cytotoxicity in various cancer cells. ${ }^{8}$ In our preliminary study, we found that GLB at nontoxic concentration could sensitize GC cells to DDP. In this paper, the sensitizing effects and possible mechanisms of GLB with alkylating agents in inhibiting GC cells are reported.

\section{Materials and methods}

\section{Reagents and cell culture}

Roswell Park Memorial Institute-1640 (RPMI-1640; Booster Biological Technology Co, Ltd, Wuhan, People's Republic of China), fetal bovine serum (Biological Industries, Beit Haemek, Israel), trypsin \& $0.02 \%$ EDTA (Gino Biopharmaceutical Technology, Hangzhou, People's Republic of China), MMC (TCI [Shanghai] Development Co., Ltd., Shanghai, People's Republic of China), CTX (Abcam,
Cambridge, UK), DDP (HaoSen, Jiangsu, People's Republic of China), and Cell Counting Kit 8 (CCK-8; Dojindo Laboratories, Kumamoto, Japan), bovine serum albumin (Amresco, Solon, OH, USA) were bought and utilized. HER-2, c-Met, p53, p27, p21, TERT, Pol $\alpha$, Pol $\varepsilon, \mathrm{Bcl} x 1$, Bax, $\beta$-Actin, GAPDH (Santa Cruz Biotechnology, Dallas, TX, USA), c-Kit, NF-кB-p65, Akt, p44/42 MAPK, PCNA, PARP, caspase 9, pro-caspase 3, cleaved caspase 3 (Cell Signaling Technology, Danvers, MA, USA), and Bcl 2 (Abcam) were used. Secondary antibodies used were antigoat, anti-rabbit, and anti-mouse (Beijing Zhongshan Golden Bridge Biotechnology Co. Ltd, Beijing, People's Republic of China). HRP substrate (Millipore Corporation, Billerica, MA, USA), skim-milk (BD-Difco, Sparks, MD, USA), MitoTracker ${ }^{\circledR}$ Red CMXRos (Invitrogen Life Technologies Corporation, Carlsbad, CA, USA), PI/RNase Staining Buffer (BD Biosciences, San Diego, CA, USA), and PEAnnexin-V apoptosis detection kit (BD Biosciences) were also purchased and used.

\section{Cell line and cell culture}

Human gastric cancer cell line SGC-7901 (purchased from ATCC, Manassas, VA, USA) was cultured in RPMI-1640 medium containing $10 \%$ heat-inactivated fetal bovine serum under adherent conditions in an atmosphere of $5 \%$ $\mathrm{CO}_{2}$ at $37^{\circ} \mathrm{C}$.

\section{Cell growth inhibition test}

The inhibition of cell growth by MMC, DDP, CTX, and GLB independently or in combinations with GLB were determined by CCK-8 assay. Various concentrations of MMC, DDP, and CTX with or without $5 \mu \mathrm{M}$ GLB concentrations were added to SGC-7901 cells for $60 \mathrm{~h}$, followed by the addition of $10 \mu \mathrm{L}$ of CCK-8 in each well; this was followed by incubation for another $1 \mathrm{~h}$. Absorbance was measured at $450 \mathrm{~nm}$ with a Bio-Rad iMark ${ }^{\mathrm{TM}}$ (Bio-Rad Laboratories, Inc., Hercules, CA, USA) Microplate Reader. The percentage of dose inhibition response was calculated using Graphpad Prism v6 (GraphPad Software, Inc., San Diego, CA, USA).

\section{Sub- $G_{\text {, }}$ analysis}

SGC-7901 cells were incubated with controls and drugs for $16 \mathrm{~h}$. Collected cells were washed twice with ice-cold PBS and were fixed overnight at $4{ }^{\circ} \mathrm{C}$ with $75 \%$ ethanol. PI/RNase staining buffer at a final concentration of $0.05 \mathrm{mg} / \mathrm{mL}$ was used to stain the cells for $15 \mathrm{~min}$ at room temperature. Cell cycle analyses were performed with BD FACSCanto ${ }^{\mathrm{TM}}$ II (BD Biosciences). 


\section{Western blot analysis}

SGC-7901 cells were treated for 16-48 $\mathrm{h}$ with the respective drugs concentrations and were subjected to phosphatase inhibitor and radioimmunoprecipitation assay lysis buffer. About $30 \mu \mathrm{g}$ of lysates were loaded to sodium dodecyl sulfate-polyacrylamide gel electrophoresis followed by blotting onto polyvinyl difluoride membrane. The membrane was blocked with $10 \%$ TBST-skim milk or 5\% BSA-TBST (for phosphoproteins) for $1 \mathrm{~h}$. For the detection of protein expressions, the membrane was incubated at $4^{\circ} \mathrm{C}$ overnight with primary antibodies (1:100-1:1,000) and HRP-conjugated secondary antibodies $(1: 2,000)$. ECL chemiluminescent HRP substrate was used to visualize immunoreactive bands. Images were developed using Bio-Rad ChemiDoc ${ }^{\mathrm{TM}}$ MP Imaging system, and protein quantification was performed using Image Lab Software v5.2.1 (Bio-Rad Laboratories, Inc.).

\section{Annexin- $\mathrm{V}$ flow cytometry analysis}

SGC-7901 cells were incubated with subsequent control and drugs at aforementioned concentrations for $24 \mathrm{~h}$. Cells were collected and washed twice with ice-cold PBS. Cells were then stained with PE-Annexin-V apoptosis detection kit and were analyzed using flow cytometry BD FACSCanto ${ }^{\mathrm{TM}}$ II (BD Biosciences).

\section{Determination of cellular reactive oxygen species}

SGC-7901 cells treated with control and GLB + drug combinations for $24 \mathrm{~h}$ were incubated with a $100 \mathrm{nM}$ final concentration of MitoTracker ${ }^{\circledR}$ Red CMXRos in RPMI-1640 for $30 \mathrm{~min}$ at growth condition appropriate for the cell growth. Cells were visualized under fluorescent microscope Lecia DM 4000 and relative fluorescence was measured with ImageJ v1.50i (National Institutes of Health, Bethseda, MD, USA).

\section{Statistical analysis}

All data in the figures are expressed as means \pm SDs and were analyzed with GraphPad by one-way analysis of variance with Tukey's $t$-tests. $P<0.05$ was considered statistically significant.

\section{Results \\ GLB significantly induces the tumoricidal effects of alkylating agents}

Our preliminary studies showed that GLB at a nontoxic concentration of $5 \mu \mathrm{M}$ sensitizes GC cells to various alkylating agents. To test the underlying mechanism of how $5 \mu \mathrm{M}$ GLB concentrations with insignificant inhibition on GC cells has sensitizing effects on the growth inhibition activity of MMC, DDP, and CTX, we first determined cytotoxic effects of MMC, DDP, and CTX with or without $5 \mu \mathrm{M}$ GLB on SGC-7901 cells. Half-maximal inhibitory concentrations $\left(\mathrm{IC}_{50 \mathrm{~s}}\right.$ ) of MMC, DDP, and CTX were $4.14 \mu \mathrm{M}, 6.01 \mu \mathrm{M}$, and $2.002 \mathrm{mM}$ to SGC-7901 cells, respectively (Figure 1A). When $5 \mu \mathrm{M}$ of GLB was used with drugs, $\mathrm{IC}_{50}$ of MMC, DDP, CTX was reduced to $1.02 \mu \mathrm{M}, 3.3 \mu \mathrm{M}, 0.96 \mathrm{mM}$ (Figure 1B). $\mathrm{IC}_{50}$ for GLB alone was $13.40 \mu \mathrm{M}$ (Figure 1A). Growth inhibition assay revealed that GLB sensitizes the tumoricidal effects
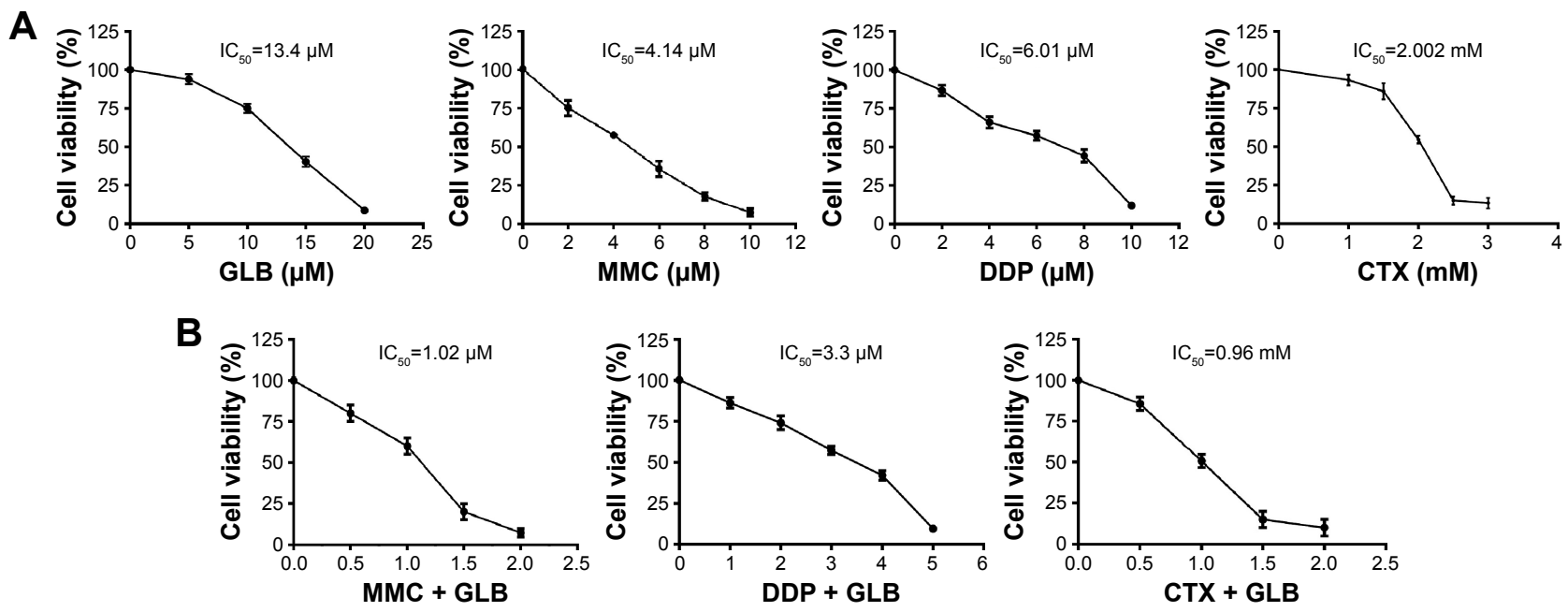

Figure I GLB significantly induces the tumoricidal effects of alkylating agents.

Notes: Effect of GLB + drugs on the proliferation of human gastric cancer cell SGC-790I. Cells were incubated with GLB, MMC, DDP, and CTX (A), and GLB + MMC, GLB + DDP, and GLB + CTX (B) for 60 h. Cell proliferation was assayed with CCK-8 and $I_{50}$ was calculated. Data presented are the means \pm SD of results from three independent experiments.

Abbreviations: CCK-8, cell counting kit-8; CTX, cyclophosphamide; DDP, cisplatin; GLB, glaucocalyxin B; IC ${ }_{50}$, half-maximal inhibitory concentration; MMC, mitomycin C. 
of MMC, DDP, and CTX up to $75.40 \% \pm 5 \%, 45.10 \% \pm 5 \%$, and $52.10 \% \pm 5 \%$, respectively. These results suggest the significant sensitization induced by GLB on the tumoricidal activities of these alkylating agents.

\section{GLB/alkylating agents combination inhibits proliferation of GC}

The tumoricidal effects of GLB + drugs combinations were found to be significantly evident and important. To unearth the underlying mechanism of how GLB sensitizes the effectiveness of these drugs in GC cells, we examined the expression of most important proteins established to have a link in GC (Figure 2). EGFR, HER-2/Neu, c-Met, and c-Kit are being used as potential targets for the treatment of GC. Decreased expression of these important target proteins upon GLB + drugs treatments suggest decreased proliferation, differentiation and lesser progression of GC as their over expression is associated with the development, progression and poor prognosis of GC. Decreased c-Kit expression suggests a decrease in the number of cells entering into a state of terminal differentiation and decreased cell survival and proliferation as well. The high c-Kit expression in GLB + DDP might lead to impaired self-renewal as the overexpression of c-Kit is associated with impaired hematopoietic stem cells. Decreased NF- $\kappa$ B-p65 cells, a subunit of NF- $\kappa B$ transcription complex is important in sustaining cell proliferation and cell survival, and defects in NF- $\kappa \mathrm{B}$ consequently increase susceptibility to cell death. Our results showed reduced expression of $\mathrm{NF}-\kappa \mathrm{B}$ in response to GLB + drugs treatments (Figure 2). Similarly, reduced Akt expression advocates the decreased proliferation and invasion of GC cells. MAPK/ERK is the downstream signaling pathway of Akt, and decreased expression of Akt leads to the decreased expression of MAPK/ERK pathways.

Expression of PCNA as an important marker for genome stability and as a component of replication and repair
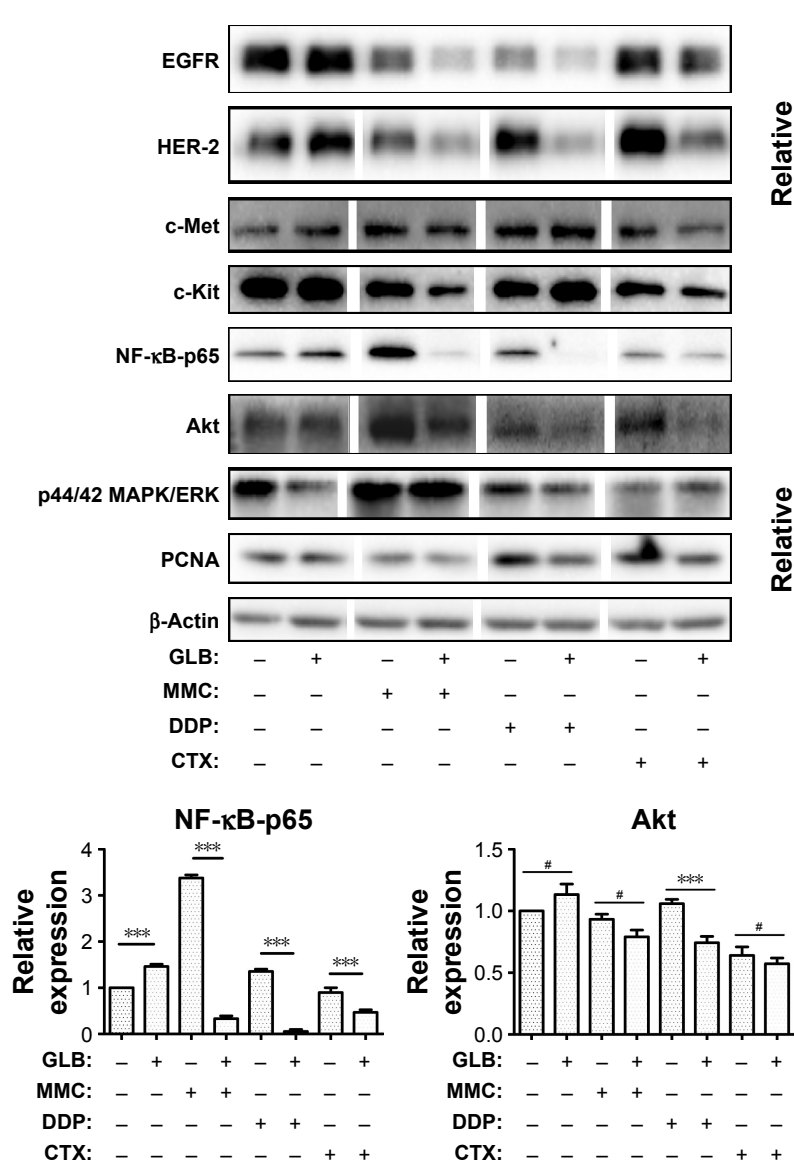

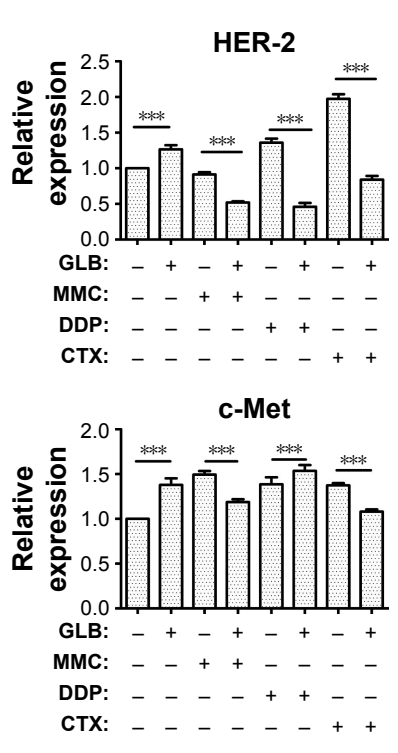

MAPK/ERK

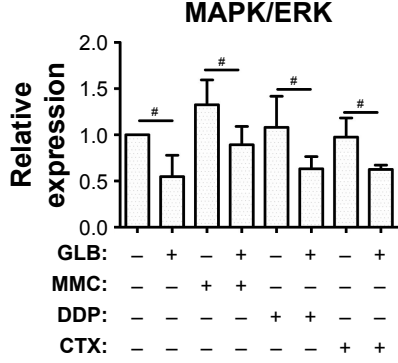

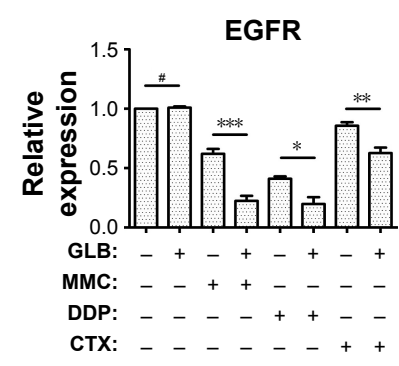

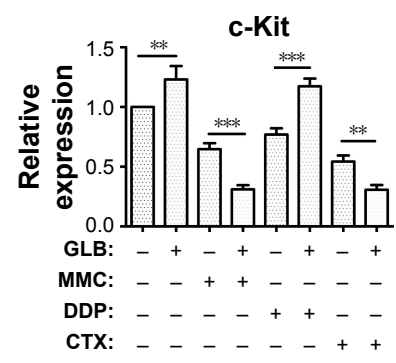

PCNA

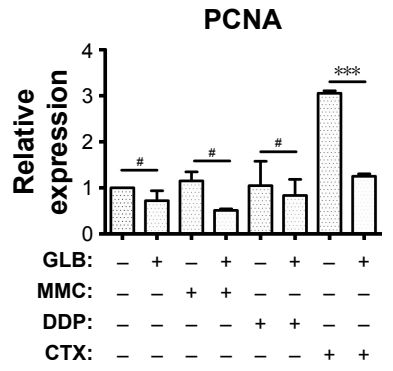

Figure $2 \mathrm{GLB} / \mathrm{alkylating} \mathrm{agents} \mathrm{inhibit} \mathrm{proliferation} \mathrm{of} \mathrm{GC}$

Notes: SGC-790I cells were treated with GLB $(5 \mu \mathrm{M})$, MMC (I.02 $\mu \mathrm{M})$, DDP $(3.3 \mu \mathrm{M}), \mathrm{CTX}(0.96 \mathrm{mM})$, GLB + MMC $(5 \mu \mathrm{M}+1.02 \mu \mathrm{M})$, GLB $+\mathrm{DDP}(5 \mu \mathrm{M}+3.3 \mu \mathrm{M})$, and $\mathrm{GLB}+\mathrm{CTX}(5 \mu \mathrm{M}+0.96 \mathrm{mM})$ for $48 \mathrm{~h}$ and protein expression level of EGFR, HER-2, c-Met, c-Kit, Akt, NF-KB, MAPK/ERK, PCNA, GAPDH, and $\beta$-Actin were analyzed with Western blotting analysis. Data presented are the mean $\pm S D$ of results from three independent experiments. $* P<0.05, * * P<0.01, * * * P<0.001$ and ${ }^{\#} P \geq 0.05$.

Abbreviations: CTX, cyclophosphamide; DDP, cisplatin; GLB, glaucocalyxin B; IC ${ }_{50}$, half-maximal inhibitory concentration; MMC, mitomycin C. 
machinery was analyzed. Upon GLB + drugs treatment, decreased expression of PCNA led to the suggestion of probable compromised proliferation and repair mechanism of GC. These outcomes suggest that GLB + drugs can significantly inhibit the proliferation, differentiation, and genome stability by targeting most important signaling pathways in GC. MMC, DDP, CTX, or GLB alone had insignificant effects at those concentrations used.

\section{GLB induces cell cycle arrest}

GC cells were treated with drugs alone or with GLB + drugs and this was followed by cell cycle analysis with flow cytometry. The results showed (Figure 3) that GLB + MMC group arrested nearly $50 \% \pm 5 \%$ of cells in $\mathrm{G}_{1}$-phase as compared with the group treated with MMC alone. GLB + DDP arrested almost $60 \% \pm 5 \%$ cells in $\mathrm{G}_{2} / \mathrm{M}$ phase as compared with control and decreased $\mathrm{S}$ phase from almost $50 \% \pm 5 \%$ to less than $10 \% \pm 5 \%$, while GLB + CTX arrested $10 \% \pm 5 \%$ more cells at the $\mathrm{G}_{0} / \mathrm{G}_{1}$ phase as compared with their corresponding control group and also caused significant $\mathrm{G}_{2} / \mathrm{M}$ or $\mathrm{G}_{0} / \mathrm{G}_{1}$ arrest. Cells treated with GLB alone showed a modest increase in $\mathrm{S}$ phase cells. The Western blot analysis revealed that treatment with GLB and GLB + drugs also supported the cell cycle analysis results as increased expression of CDK inhibitors p21 and p27, and decrease expression level of p53 and CD1 were observed. These results are consistent with the results that GLB + drugs inhibit cell proliferation and cell division.

\section{GLB induces damage to DNA replication machinery}

Diterpenes treatment in cancer cells may lead to telomerase shortening, which can lead to apoptosis. So, we examined the level of TERT in control and GLB + drugs-treated cells. Results showed (Figure 4) that GLB itself facilitates the expression of TERT, but when used in combination with other drugs it led to decreased TERT expression. We assumed that GLB + drugs could damage the DNA and DNA repair machinery. To test this hypothesis, we observed the expression of two vital enzymes, DNA polymerase alpha $($ pol $\alpha$ ) involved in the initiation of DNA replication and DNA polymerase epsilon $(\mathrm{Pol} \varepsilon)$ in the replication of leading strand. Our results exhibited that GLB + drugs considerably constrained the expression of these two important enzymes necessary for DNA replication. This decrease may lead to decreased proliferation or can induce apoptosis.

A
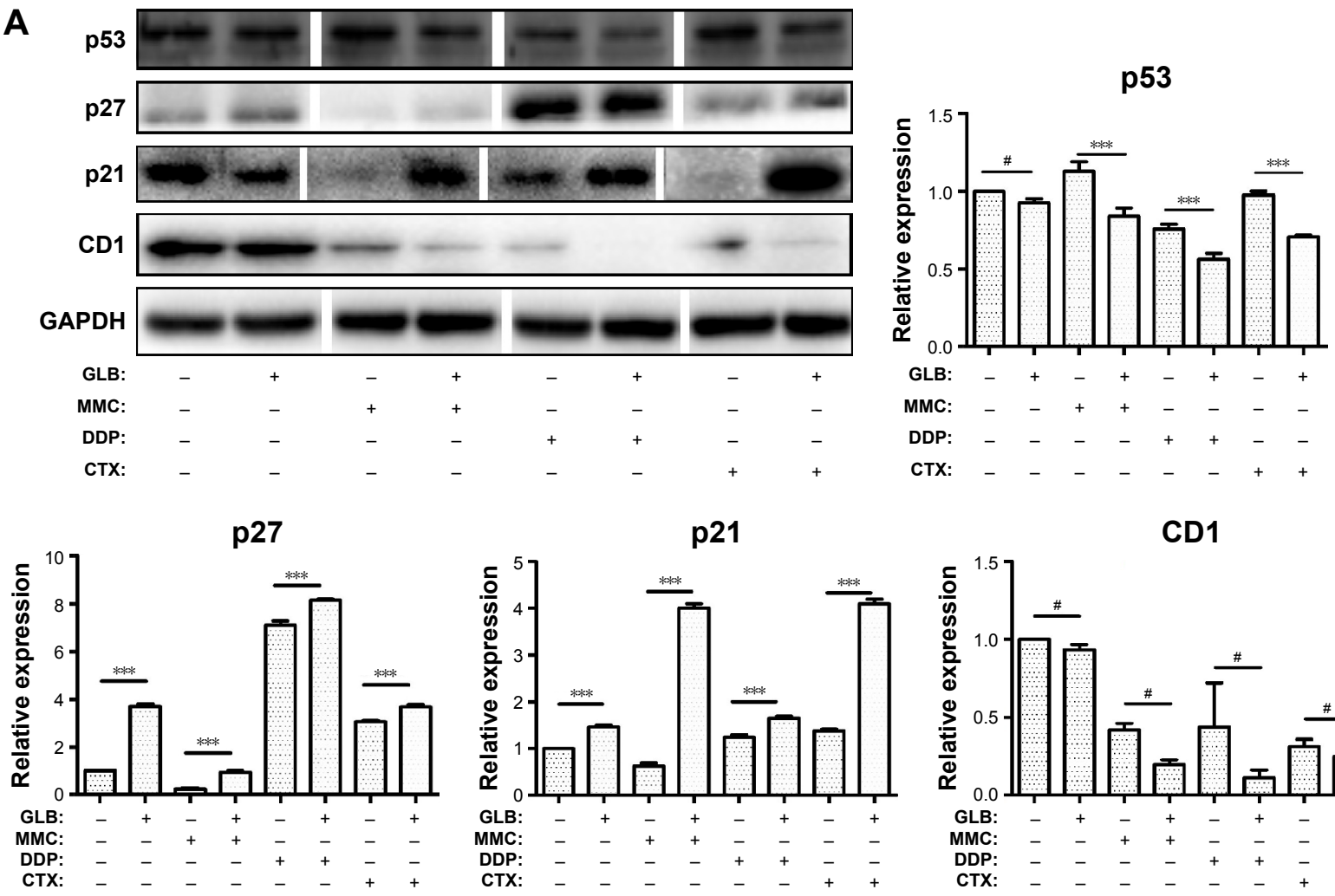

p21

CD1

Figure 3 (Continued)
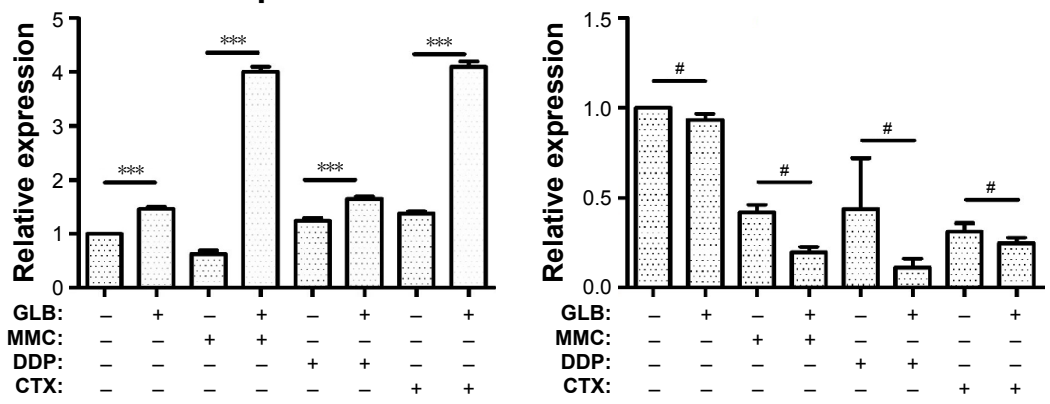

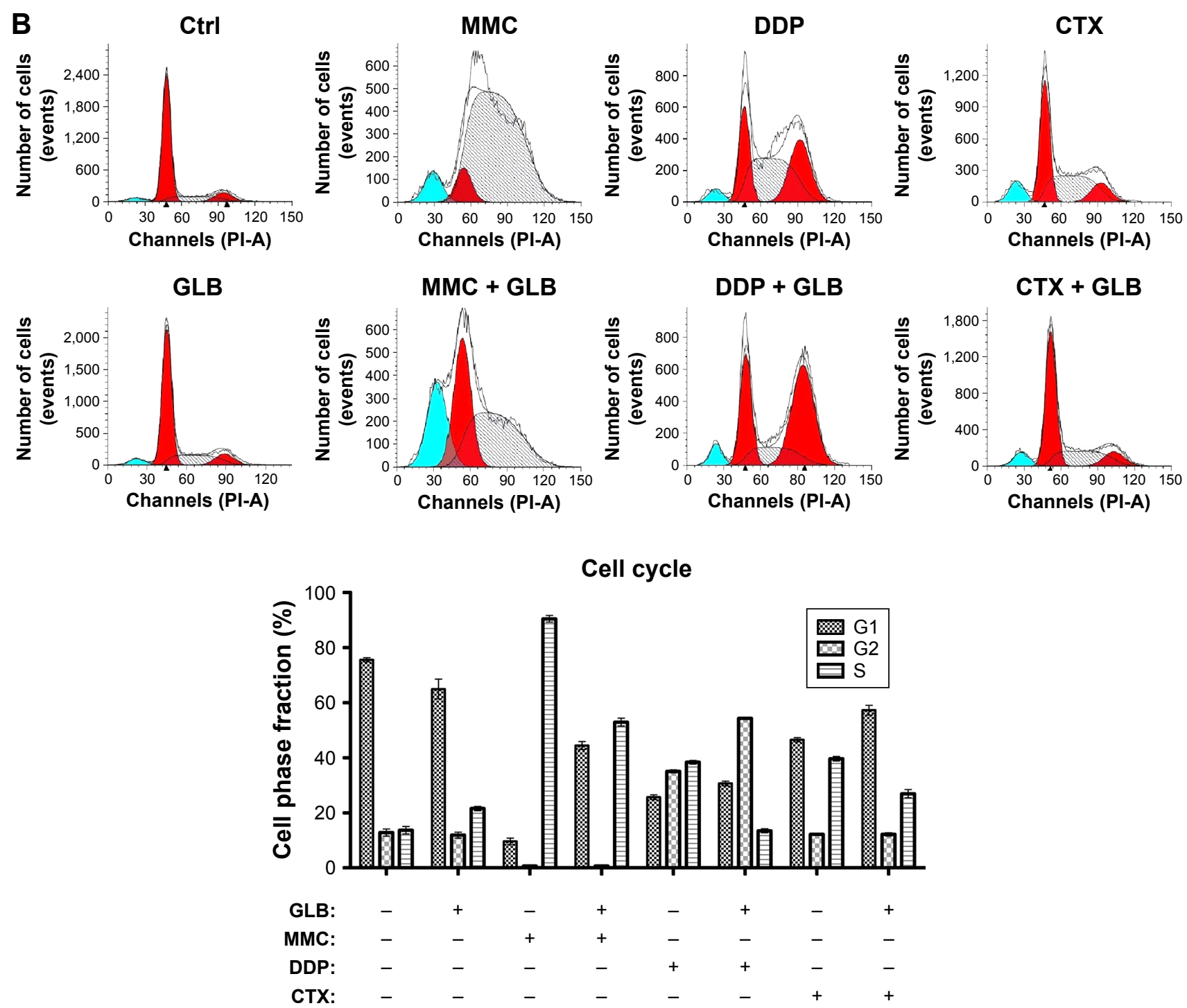

Figure 3 GLB induces cell cycle arrest.

Notes: (A) SGC-790I cells were treated with GLB $(5 \mu \mathrm{M})$, MMC (I.02 $\mu \mathrm{M})$, DDP $(3.3 \mu \mathrm{M}), \mathrm{CTX}(0.96 \mathrm{mM}), \mathrm{GLB}+\mathrm{MMC}(5 \mu \mathrm{M}+\mathrm{I} .02 \mu \mathrm{M}), \mathrm{GLB}+\mathrm{DDP}(5 \mu \mathrm{M}+3.3 \mu \mathrm{M})$, and GLB $+C T X(5 \mu M+0.96 \mathrm{mM})$ for $16 \mathrm{~h}$ and protein expression levels of p53, p27, p2 I, CDI were analyzed. (B) Representative histograms from flow cytometry of cell cycle analysis in SGC-790I human GC cells treated with GLB + drugs. Data presented are the mean \pm SD of results from three independent experiments. $* * * P<0.00 \mathrm{I}$ and \#P $\geq 0.05$.

Abbreviations: CTX, cyclophosphamide; DDP, cisplatin; GLB, glaucocalyxin B; IC , half-maximal inhibitory concentration; MMC, mitomycin C.

\section{GLB sensitize GC cells to apoptosis}

Diminished NF- $\kappa B$ expression, telomerase activity, Pol $\alpha$, and Pol $\varepsilon$ led to the assumption that GLB + drugs could induce apoptosis in GC. We tested the expression analysis of most important markers for apoptosis, (Figure 5A). Activated PARP/caspase 9/caspase 3 pathway and increased expression of cleaved caspase 3 demonstrated that GLB + drugs activated caspase/PARP pathway. We further determined the level of Bcl 2, Bax, and Bcl xl in SGC-7901 cells upon $\mathrm{GLB}+$ drugs treatments. The ratio of $\mathrm{Bax}$ to $\mathrm{Bcl} 2$ increased significantly in cells treated with GLB + drugs, compared with the controls. These results recommend that GLB + drugs treatment not only induces apoptosis by triggering caspase but also by regulating the expression of apoptosis-associated proteins $\mathrm{Bcl}$ 2, Bax, and $\mathrm{Bcl} x \mathrm{xl}$ (Figure 5A).

The apoptotic effects of GLB + drugs on SGC-7901 cells by flow cytometry are shown in Figure 5B. The number of cells in late apoptosis stage were $53.5 \% \pm 5 \%, 48.9 \% \pm 5 \%$, and $20 \% \pm 5 \%$ for GLB/MMC, GLB/DDP and GLB/CTX, while for their matching controls, GLB, MMC, DDP, and CTX, it was with $10.8 \% \pm 5 \%, 7.2 \% \pm 5 \%, 9.4 \% \pm 5 \%$, and $7.1 \% \pm 5 \%$, respectively, suggesting the significant apoptosis potential of GLB + drugs on GC cells. We further examined if the increased production of reactive oxygen species 

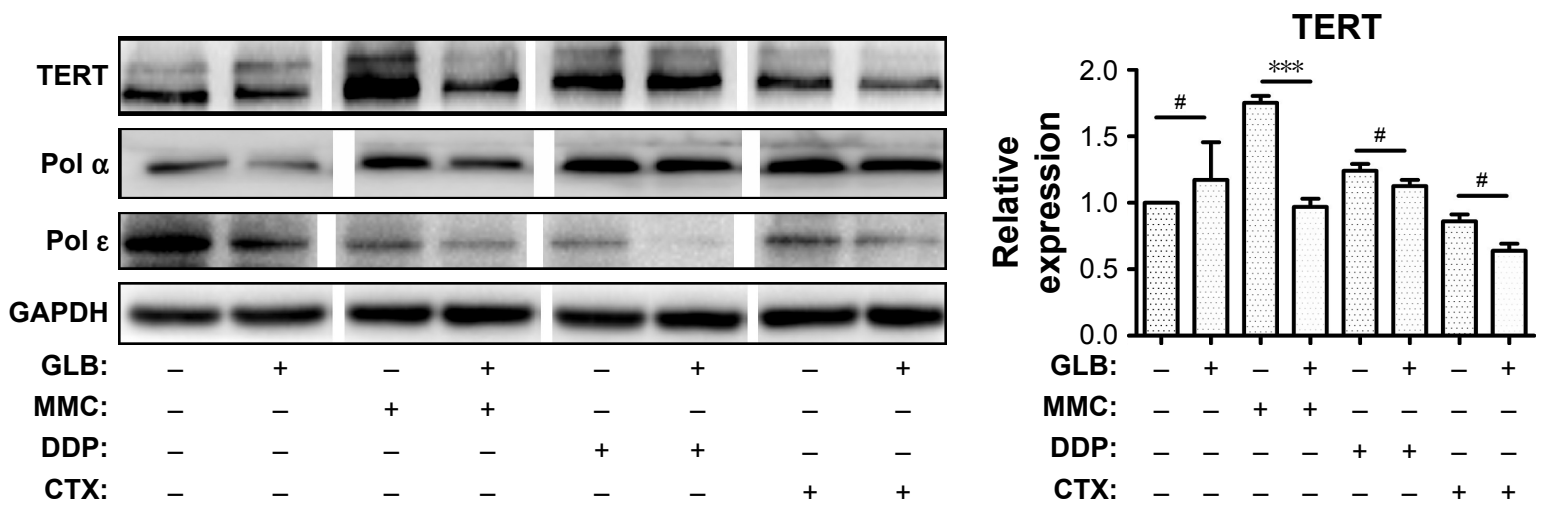

Pol $\alpha$

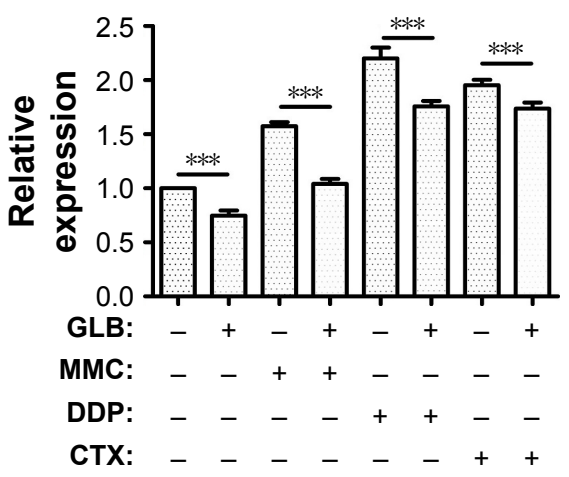

Pol $\varepsilon$

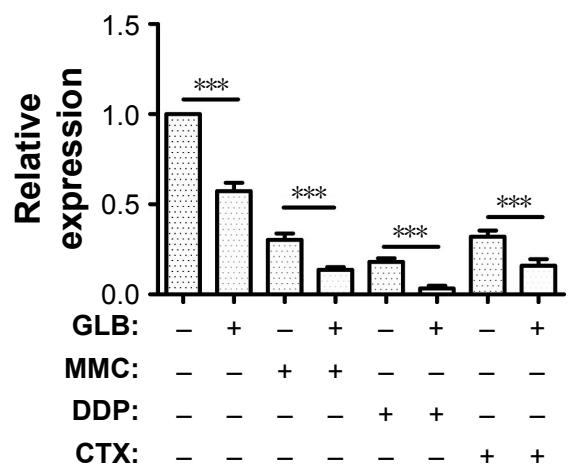

Figure 4 GLB induces damage to DNA replication machinery.

Notes: SGC-790I cells were treated with GLB $(5 \mu \mathrm{M})$, MMC (I.02 $\mu \mathrm{M})$, DDP $(3.3 \mu \mathrm{M})$, CTX $(0.96 \mathrm{mM}), \mathrm{GLB}+\mathrm{MMC}(5 \mu \mathrm{M}+\mathrm{I} .02 \mu \mathrm{M})$, GLB + DDP $(5 \mu \mathrm{M}+3.3 \mu \mathrm{M})$, and GLB + CTX $(5 \mu M+0.96 \mathrm{mM})$ for $24 \mathrm{~h}$ and protein expression levels of TERT, Pol $\alpha$, and Pol $\varepsilon$ and GAPDH as a control were measured. Data presented are the mean \pm SD of results from three independent experiments. $* * * P<0.001$ and ${ }^{\#} P \geq 0.05$.

Abbreviations: CTX, cyclophosphamide; DDP, cisplatin; GLB, glaucocalyxin B; IC ${ }_{50}$, half-maximal inhibitory concentration; MMC, mitomycin C.

(ROS) had some effect on apoptosis. The results showed a $60 \% \pm 5 \%, 30 \% \pm 5 \%$, and $150 \% \pm 5 \%$ increase in the level of ROS in response to GLB + MMC, GLB + DDP, and GLB + CTX treatments as compared with corresponding MMC, DDP, and control groups, respectively (Figure 5C). These results suggest that $\mathrm{GLB} /$ drugs can induce apoptosis in $\mathrm{GC}$ cells by activating ROS.

LC3A/B-II expression analysis showed a significant increase in the expression of this autophagy marker. 100\% $\pm 5 \%$ and $400 \% \pm 5 \%$ increase in LC3A/B-II expression level upon $\mathrm{GLB}+\mathrm{MMC}$ and GLB + DDP treatments were observed, while the changes were insignificant upon GLB + CTX treatment.

\section{Discussion}

GLB is a highly liposoluble compound, which enables its easier infiltration into the cells. ${ }^{9}$ GLB belongs to the class diterpenes and can function either by inhibiting NF- $\kappa \mathrm{B}$ transcriptional activity or by inhibiting telomerase activity, leading to growth arrest and apoptosis..$^{10,11}$

MMC, DDP, and CTX are most extensively used chemotherapeutic agents and can inhibit the tumor growth effectively, yet they have certain life-threatening side effects. They belong to the drug class of interstrand cross-links (ICLs) that prevent transcription and replication by inhibiting DNA strand separation. These agents cross-link DNA with proteins or, alternatively, cross-link two DNA bases within the same DNA strand (intra-strand cross-links) or on opposite DNA strand (ICLs). ${ }^{12}$ The ICL agents were one of the earliest, and are still the most widely used, chemotherapeutic drugs. The main setbacks in cancer treatment are either the intrinsic or the acquired resistance developed by tumors against the drugs or the limitations of anticancer compounds owing to their life-threatening adverse and nontargeted toxicities. While the underlying mechanisms of these side effects remain incompletely known, the severity of these side effects can be reduced by using combination therapies that have the effect of lessening the overall dose of each single agent. In addition, sensitizing combinations with nonoverlapping toxicities can reduce side effects.

It was found that GLB alone inhibits the growth of SGC-7901 with an $\mathrm{IC}_{50} 13.40 \mu \mathrm{g} / \mathrm{mL}$. Results further indicate that inclusion of $5 \mu \mathrm{M}$ GLB in MMC, DDP, and CTX 

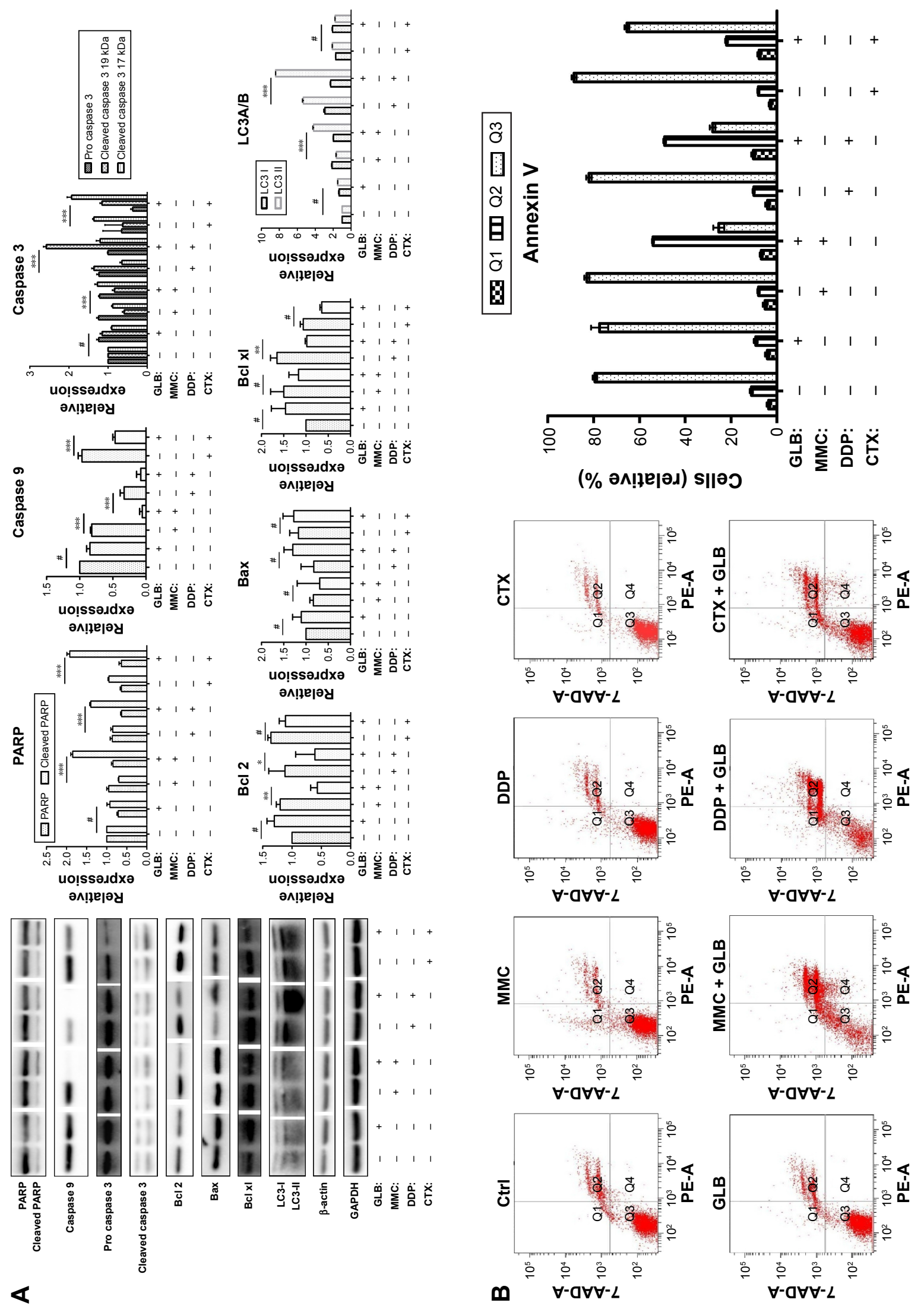

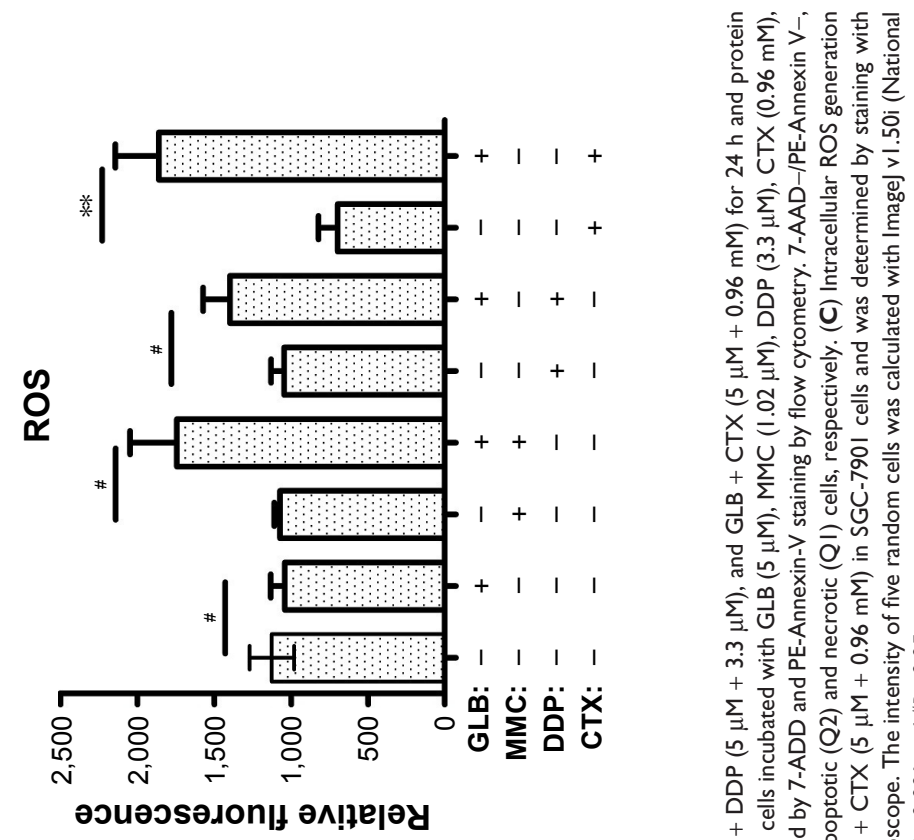

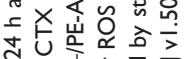

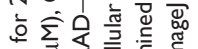
$\sum_{\varepsilon}^{m}$

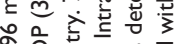

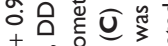
$\sum_{3} \hat{\bar{\Sigma}}$

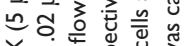
U

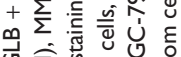

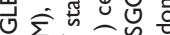
o

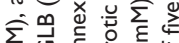

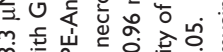
T+

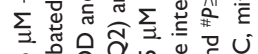

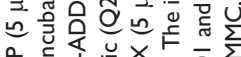

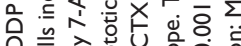

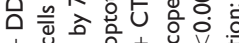
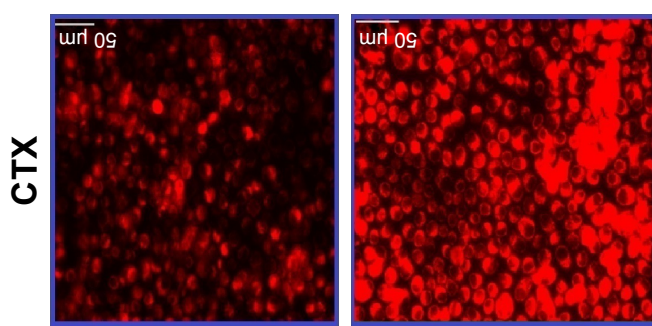

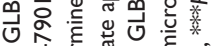

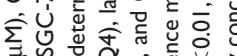

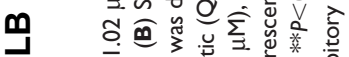
0 广 +

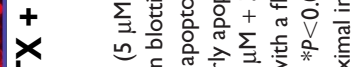
ํㅡㄴ

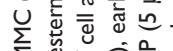

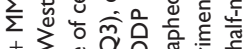

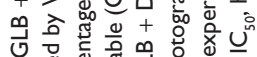
U d

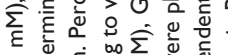

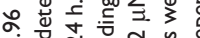

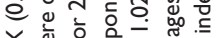
$\times$

吕
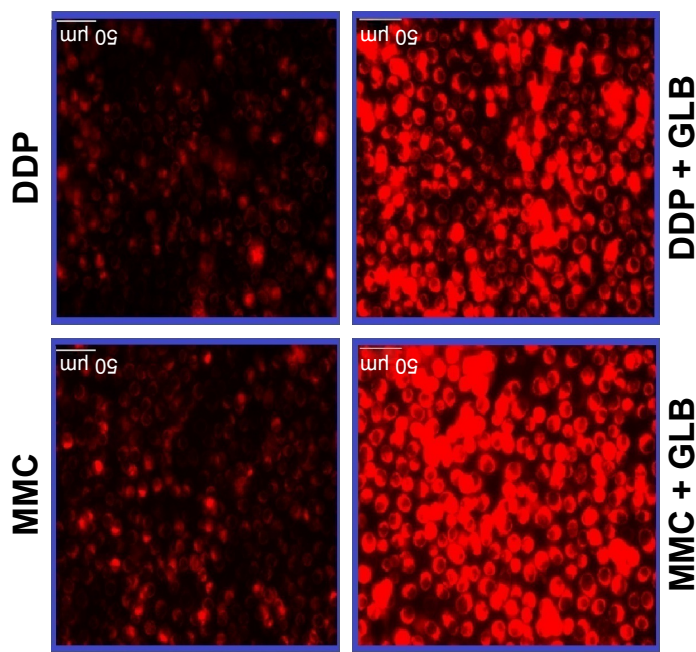

है।

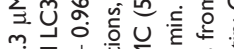

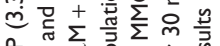

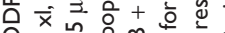

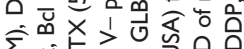

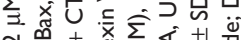
סิ

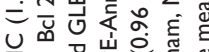

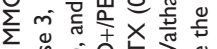

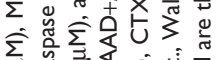

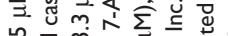

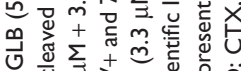
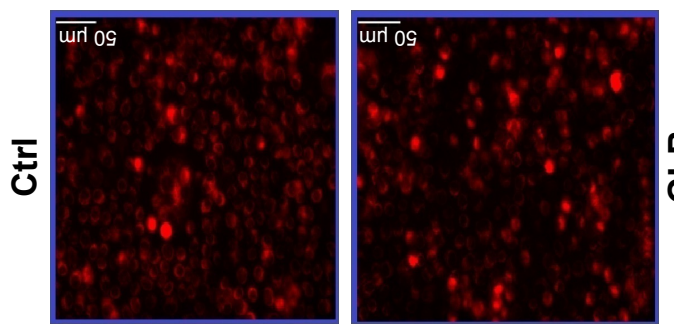

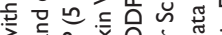

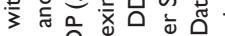

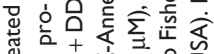

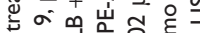

当

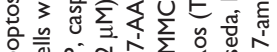

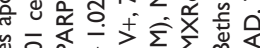

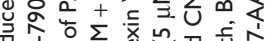

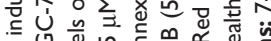

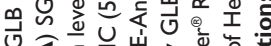

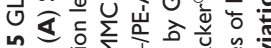

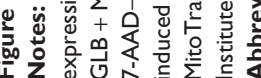


significantly reduced the effective dose essential to produce that effect. These results signify that GLB can sensitize the tumoricidal ability of these DNA binding agents. The results obtained suggest that GLB inclusion might lead to reduced side effects due to the reduced effective dosage of those drugs required in treatment.

We observed that the combination of GLB with these drugs resulted in the decreased expression of key proteins involved in GC cell proliferation, survival, migration, and GC progression. EGFR overexpression is associated with poor prognosis, thus providing a potential therapeutic target for GC..$^{13}$ Similarly, HER-2 signal transduction inhibition suppresses cell growth in GC. ${ }^{14} \mathrm{C}-$ Met promotes GC cell proliferation, migration, and survival, ${ }^{15}$ whereas c-Kit maintains cell proliferation and differentiation and NF- $\mathrm{\kappa B}$ is involved in the development, maintenance, and progression of cell adhesion, differentiation, and apoptosis. ${ }^{16,17}$ MAPK pathway plays a crucial role in cell proliferation control. Sustained activation of ERK1/ERK2 in normal cells is necessary for $\mathrm{G}_{1}$ to S-phase progression, and thus has a positive association as a cell cycle regulator. ${ }^{18}$ Akt regulates cell proliferation, survival, invasion, angiogenesis, and cell size. ${ }^{19}$ PCNA for genome stability and as a component of replication and repair machinery has earned a reputation as the "ringmaster" or "maestro" of the genome. ${ }^{20}$ PCNA also regulates entry into S-phase via interactions with the cell cycle regulator p21. ${ }^{21}$ These results showed that GLB + drugs combination can decrease the expression of all these key regulators of proliferation, invasion, migration, and progression, and thus inhibit the growth of GC cells in vitro.

We further analyzed the effects of GLB combined with drugs on cell cycle. The results showed (Figure 3) that GLB + MMC arrested cells in $\mathrm{G}_{1}$-phase, GLB + DDP arrested cells in $\mathrm{G}_{2} / \mathrm{M}$ while GLB + CTX arrested cells in $\mathrm{G}_{0} / \mathrm{G}_{1}$ phase as compared with their corresponding GLB, MMC, DDP and CTX control groups. p53 regulates the progression of cell cycle negatively in response to cellular stress or DNA damage. Transcription of p53 is activated during cells' progression from $\mathrm{G}_{0} / \mathrm{G}_{1}$ into S-phase. ${ }^{22}$ Decreased expression of $\mathrm{p} 53$ leads to decreased number of cells in S-phase, which is consistent with the cell cycle analysis findings. Moller proposed that p27 showed negative regulation of $\mathrm{CD} 1$, which is consistent with the result of this study. ${ }^{23}$ Following DNA damage, CDK inhibitor p21 is activated and induces cell cycle arrest by inhibiting DNA replication and apoptosis. ${ }^{24}$ The data presented in Figure 3 show that increased expression of p21 in $\mathrm{GLB}$ + drug-treated groups is responsible for cell cycle arrest and other DNA damages. CD1 overexpression is linked to the progression and development of cancer. ${ }^{25}$ In this study, decreased CD1 expression suggests that GLB + drugs can halt progression and development of cancer.

Replication of DNA is a precise and dynamic process and DNA polymerases, $\mathrm{Pol} \alpha$ and $\mathrm{Pol} \varepsilon$, function in collaboration to facilitate efficient and high-fidelity genome replication. Pol $\alpha$ polymerase is essential for chromosomal replication, and Pol $\varepsilon$ is required for chromosomal replication. ${ }^{26}$ The decrease in expression of both Pol $\alpha$ and Pol $\varepsilon$ upon GLB + drugs treatment suggests impaired DNA replication and telomerase shortening. Impaired DNA replication and telomerase shortening can lead to apoptosis.

Apoptosis is inevitable for the proper homeostatic maintenance and survival. Caspase is one of the most important signaling cascades associated with apoptosis. Caspases are usually present in an inactive zymogen form. Activated caspase cleaves and activates effector caspases, which can then drive apoptosis. ${ }^{27}$ Cleavage of PARP by caspase is considered a hallmark of apoptosis.

In this study, GLB + drugs resulted in increased apoptotic rate compared with controls (Figure 5A). Activated caspase 9, an initiator caspase, cleaved the effector caspase 3, while PARP was cleaved into cleaved PARP. These results support the hypothesis that the sensitizing tumoricidal effects of GLB + drugs contribute greatly in inducing apoptosis, more so than controls. Moreover, GLB + drugs limited the antiapoptotic factor $\mathrm{Bcl} 2$ and stimulated the expression of proapoptotic factor Bax (Figure 5A). Results showed that ROS generation is also involved in the trigger of apoptosis, as proposed by Chen et al, ${ }^{28}$ who demonstrated that increased ROS production could trigger apoptosis. Similarly, the flow cytometry analysis also consolidated the apoptotic effects associated with GLB + drugs treatment.

LC3s (MAP1-LC3A, B, and C) are structural proteins of autophagosomal membranes and are used as autophagy markers. The presence of LC3 in autophagosomes and the conversion of LC3-I to the lower migrating form LC3-II have been used as indicators of autophagy. ${ }^{29}$ As can be seen from Figure 5, increased autophagy in response to GLB + $\mathrm{MMC}$ and GLB + DDP treatment was observed to $100 \%$ and $400 \%$, respectively, while insignificant autophagy effects were found in GLB + CTX group. Increased autophagy can also be the reason for increased cell death. Taken together, these results suggest that GLB + drugs treatment promotes GC cell death by both activating caspase and regulating the expression of $\mathrm{Bcl} 2$ family proteins and by autophagy.

\section{Conclusion}

In conclusion, this study demonstrates that GLB can improve MMC-, DDP-, and CTX-based chemotherapy in GC by 
decreasing the dose required, which in turn can reduce the side effects associated with MMC, DDP, and CTX. GLB sensitizes GC cells by interfering with proliferation, arresting the cell cycle, impairing replication, leading to telomerase shortening, and promoting apoptosis. Further in vivo investigation with tumor xenograft animal models will provide us more information on the potential combination therapy of GLB with MMC, DDP, or CTX in clinical treatment of GC. Moreover, identification of the direct targeting molecules of GLB and alkylating agents will provide us a better understanding of the sensitization effect.

\section{Acknowledgment}

The work is supported by the National Natural Science Foundation of China (grant number 81172516).

\section{Disclosure}

The authors report no conflicts of interest in this work.

\section{References}

1. Rahman R, Asombang AW, Ibdah JA. Characteristics of gastric cancer in Asia. World J Gastroenterol. 2014;20(16):4483-4490.

2. Volpato M, Seargent J, Loadman PM, Phillips RM. Formation of DNA interstrand cross-links as a marker of Mitomycin C bioreductive activation and chemosensitivity. Eur J Cancer. 2005;41(9):1331-1338.

3. Perrone F, De Placido S, Carlomagno C, et al. Mitomycin C and Mitoxantrone in anthracycline-pretreated advanced breast cancer patients: a phase II study. Am J Clin Oncol. 1994;17(3):218-222.

4. Ali IA, Wani W, Saleem K, Haque A. Platinum compounds: a hope for future cancer chemotherapy. Anti-Cancer Agent Med Chem (Formerly Curr Med Chem-Anti-Cancer Agents). 2013;13(2):296-306.

5. Sastry J, Kellie SJ. Severe neurotoxicity, ototoxicity and nephrotoxicity following high-dose cisplatin and amifostine. Pediat Hematol Oncol. 2005;22(5):441-445.

6. Bass KK, Mastrangelo MJ. Immunopotentiation with low-dose cyclophosphamide in the active specific immunotherapy of cancer. Cancer Immunol Immunother. 1998;47(1):1-12.

7. Sun HD, Huang SX, Han QB. Diterpenoids from Isodon species and their biological activities. Nat Prod Rep. 2006;23(5):673-698.

8. Gao LW, Zhang J, Yang WH, Wang B, Wang JW. Glaucocalyxin A induces apoptosis in human leukemia HL-60 cells through mitochondriamediated death pathway. Toxicol In Vitro. 2011;25(1):51-63.

9. Gan P, Zhang L, Chen Y, et al. Anti-inflammatory effects of glaucocalyxin B in microglia cells. J Pharmacol Sci. 2015;128(1):35-46.

10. Leung CH, Grill SP, Lam W, Han QB, Sun HD, Cheng YC. Novel mechanism of inhibition of nuclear factor- $\mathrm{\kappa B}$ DNA-binding activity by diterpenoids isolated from Isodon rubescens. Mol Pharmacol. 2005;68(2):286-297.
11. Buys CH. Telomeres, telomerase, and cancer. N Engl J Med. 2000; 342(17):1282-1283.

12. O'Connor PM, Kohn KW. Comparative pharmacokinetics of DNA lesion formation and removal following treatment of L1210 cells with nitrogen mustards. Cancer Commun. 1990;2(12):387-394.

13. Okines A, Cunningham D, Chau I. Targeting the human EGFR family in esophagogastric cancer. Nat Rev Clin Oncol. 2011;8(8):492-503.

14. Wainberg ZA, Anghel A, Desai AJ, et al. Lapatinib, a dual EGFR and HER2 kinase inhibitor, selectively inhibits HER2-amplified human gastric cancer cells and is synergistic with trastuzumab in vitro and in vivo. Clin Cancer Res. 2010;16(5):1509-1519.

15. Lordick F. Targeting the HGF/MET pathway in gastric cancer. Lancet Oncol. 2014;15(9):914-916.

16. Chen S, Xiong A. The progress and implication of stem cell factor. Basic Med Sci Clin. 2002;22(5):385-390.

17. Gupta SC, Sundaram C, Reuter S, Aggarwal BB. Inhibiting NF-кB activation by small molecules as a therapeutic strategy. BBA Gene Regul Mech. 2010;1799(10):775-787.

18. Meloche S, Pouysségur J. The ERK1/2 mitogen-activated protein kinase pathway as a master regulator of the G1-to S-phase transition. Oncogene. 2007;26(22):3227-3239.

19. Altomare DA, Testa JR. Perturbations of the AKT signaling pathway in human cancer. Oncogene. 2005;24(50):7455-7464.

20. Moldovan GL, Pfander B, Jentsch S. PCNA, the maestro of the replication fork. Cell. 2007;129(4):665-679.

21. Warbrick E, Lane DP, Glover DM, Cox LS. A small peptide inhibitor of DNA replication defines the site of interaction between the cyclindependent kinase inhibitor p21 WAF1 and proliferating cell nuclear antigen. Curr Biol. 1995;5(3):275-282.

22. Reisman D, Takahashi P, Polson A, Boggs K. Transcriptional regulation of the p53 tumor suppressor gene in S-phase of the cell-cycle and the cellular response to DNA damage. Biochem Res Int. 2012;2012:808934.

23. Møller MB. P27 in cell cycle control and cancer. Leuk Lymphoma. 2000;39(1-2):19-27.

24. Cazzalini O, Scovassi AI, Savio M, Stivala LA, Prosperi E. Multiple roles of the cell cycle inhibitor p21 CDKN1A in the DNA damage response. Mutat Res/Rev Mutat Res. 2010;704(1):12-20.

25. Alao JP. The regulation of cyclin D1 degradation: roles in cancer development and the potential for therapeutic invention. Mol Cancer. 2007;6(1):24-39.

26. Foiani M, Marini F, Gamba D, Lucchini G, Plevani P. The B subunit of the DNA polymerase alpha-primase complex in Saccharomyces cerevisiae executes an essential function at the initial stage of DNA replication. Mol Cell Biol. 1994;14(2):923-933.

27. Li J, Yuan J. Caspases in apoptosis and beyond. Oncogene. 2008; 27(48):6194-6206.

28. Chen W, Zhao Z, Li L, et al. Hispolon induces apoptosis in human gastric cancer cells through a ROS-mediated mitochondrial pathway. Free Radical Biol Med. 2008;45(1):60-72.

29. Kabeya Y, Mizushima N, Yamamoto A, Oshitani-Okamoto S, Ohsumi Y, Yoshimori T. LC3, GABARAP and GATE16 localize to autophagosomal membrane depending on form-II formation. J Cell Sci. 2004; 117(13):2805-2812

\section{Publish your work in this journal}

Drug Design, Development and Therapy is an international, peerreviewed open-access journal that spans the spectrum of drug design and development through to clinical applications. Clinical outcomes, patient safety, and programs for the development and effective, safe, and sustained use of medicines are the features of the journal, which

\section{Dovepress}

has also been accepted for indexing on PubMed Central. The manuscript management system is completely online and includes a very quick and fair peer-review system, which is all easy to use. Visit http://www.dovepress.com/testimonials.php to read real quotes from published authors. 